\title{
"CHRONOTRON" TIMING DETECTORS FOR EAS STUDIES
}

\author{
Aliya Baktoraz, ${ }^{a, *}$ Nurzhan Saduyev,${ }^{a, b}$ Orazaly Kalikulov, ${ }^{a}$ Dmitriy Beznosko, ${ }^{c}$ \\ Yerzhan Mukhamejanov, ${ }^{d}$ Shynbolat Utey, ${ }^{a}$ Saken Shinbulatov, ${ }^{a}$ Nurzhan \\ Yerezhep, ${ }^{a}$ Askhat Zhumabayev, ${ }^{a}$ Valeriy Zhukov, ${ }^{b}$ Alexander Shepetov ${ }^{b}$ \\ a al-Farabi Kazakh National University, \\ 050040, al-Farabi, 71, Almaty, Kazakhstan \\ ${ }^{b}$ P.N. Lebedev Physical Institute of the Russian Academy of Sciences, \\ 119991, Leninsky avenue, 53, Moscow, Russia \\ ${ }^{c}$ Clayton State University, University System of Georgia, \\ 2000 Clayton State Blvd, Morrow, GA 30260, USA \\ ${ }^{d}$ Joint Institute for Nuclear Research, \\ 141980, str. Joliot-Curie, 6, Dubna, Russia
}

E-mail: a_baktoraz@mail.ru

\begin{abstract}
The extensive air showers (EAS) detector system consisting of timing detection is being built for the reconstruction of the EAS axis direction using chronotron timing information. This system consists of eight scintillator-based individual detectors $(100 \times 100 \times 1 \mathrm{~cm})$ using wavelength shifting fibers for light collection ("Chronotron" installation). The goal of the project is to supplement a complex of EAS installations that is located at the elevation of $3340 \mathrm{~m}$ above sea level at the (Tien Shan High-altitude Scientific Station) TSHSS near the city of Almaty, Kazakhstan, with the system of detectors with fast timing. This work presents the current design, the characteristics from the simulation and the performance of the prototype.
\end{abstract}

37th International Cosmic Ray Conference (ICRC 2021)

July 12th-23rd, 2021

Online-Berlin, German

\section{*Presenter}

(C) Copyright owned by the author(s) under the terms of the Creative Commons 


\section{Introduction}

EAS are secondary cosmic rays resulting from the interaction of primary cosmic rays with the Earth's atmosphere. At TSHSS there are several installations for the study of EAS: Horizon$\mathrm{T}$ installation [1], HADRON-55, burst detector etc.

The purpose of the 'Chronotron' installation is to determine the axis direction of EAS. Registration with eight detectors will make it possible to consider the effect of fluctuations of the EAS front when determining the zenith and azimuthal angles when analyzing time surfaces by the minimization method.

\section{Chronotron timing detectors}

Chronotron timing detectors are used to investigate the array angles (zenith and azimuthal angles) of EAS. Scintillation detectors using wavelength shifting fibers for light collection are the working body of Chronotron installation, with their relative positions schematic shown in Figure 1. Each scintillation detector has a shape $1000 \times 1000 \times 10 \mathrm{~mm}$ (Figure 2). The scintillation light is collected by the WLS fibers and then detected by FEU-115M photomultiplier tubes [2]. The signals from PMT were analyzed using a CAEN DT 57258 Channel (14 bit 500 MS/s) digitizer [3].

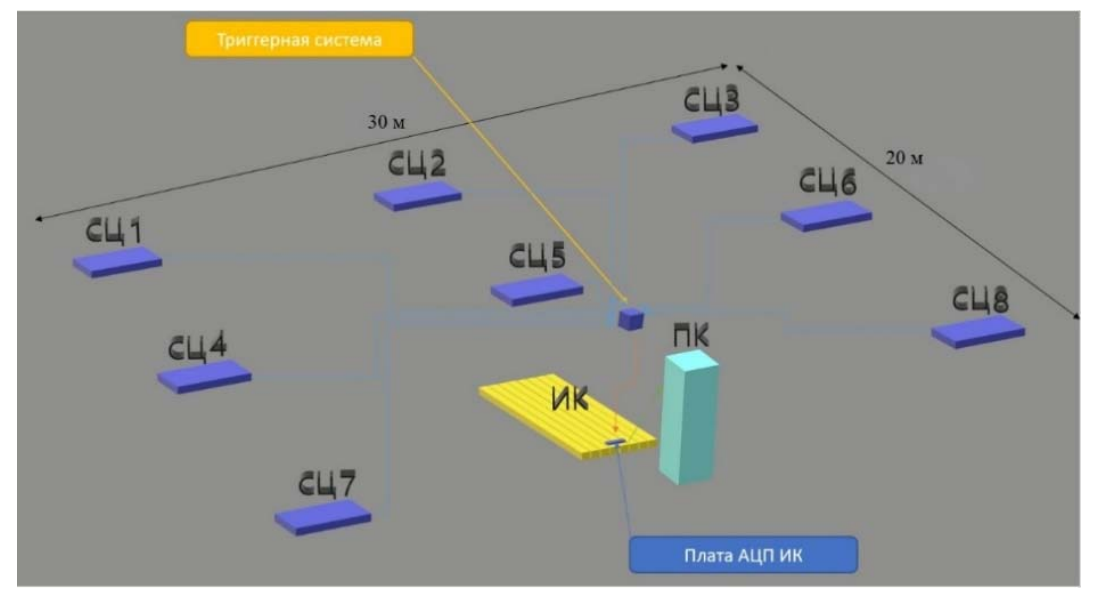

Figure 1 - The location of scintillation detectors 


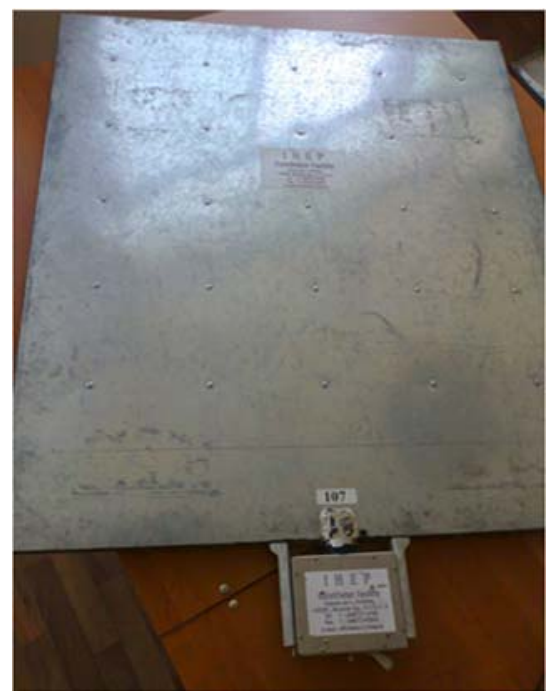

Figure 2 - General view of the scintillation detector

\section{Zenith and azimuthal angles measurement method}

To obtain the zenith and azimuthal angles, we will use the coordinates of the location of each detector. Plane equation can be written as follows:

$$
a x+b y+c z+d=0
$$

where $a, b, c$ - coordinates of the normal vector, $a^{2}+b^{2}+c^{2}=1$. In our task $z=0$, because detectors are located on the same level. In the approximation that the shower front is flat we can use formula (1). The distance from the ith detector with coordinates $\mathrm{x}_{\mathrm{i}}, \mathrm{y}_{\mathrm{i}}$ and $\mathrm{z}_{\mathrm{i}}$ to the shower plane is:

$$
\delta_{i}=a x_{i}+b y_{i}+c z_{i}+d
$$

where $\delta_{i}=t_{i} * c, t i$ - is the relative response time of the $i$ detector, $c$-speed of light [4]. Then, using the least squares method and Cramer method, we will find the coefficients a, b, c. Thus, arrival angles of shower are determined as follows:

\section{First experimental results}

$$
\begin{gathered}
\theta=\arccos (c) \\
\varphi=\operatorname{arctg}(b / a)
\end{gathered}
$$

A preliminary single-particle calibration of one SC detector was carried out. The signal rise time is defined as the time interval between the $10 \%$ and the $50 \%$ of the peak area, and the total width as the time interval between $10 \%$ and $80 \%$. The reason for these definitions is to clearly define the start of the pulse above any noise floor, to have a median of the area as a definition of the 'center' and to cut out the possible long tails from the cables (Figure 3). 

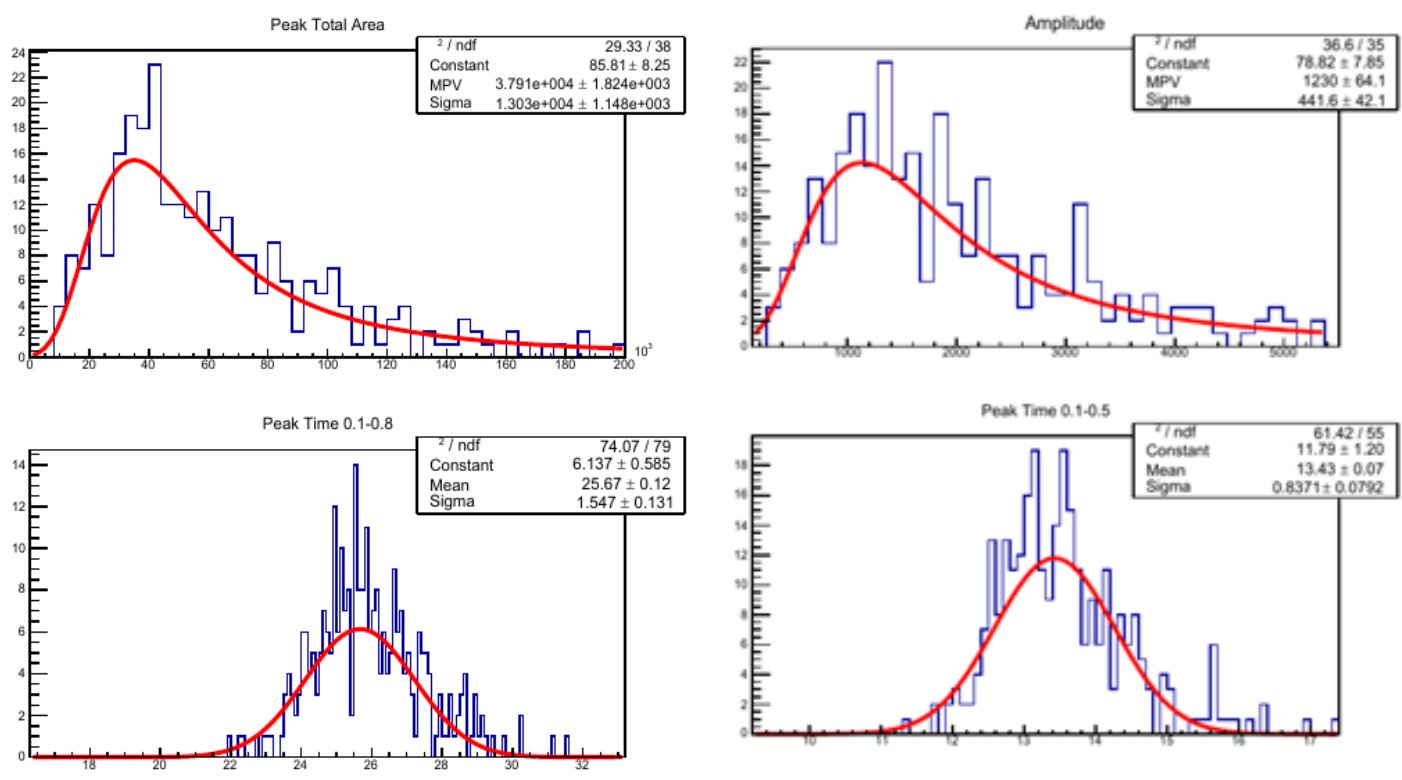

Figure 3 - Single-particle calibration SC detector

The figure 4 shows one event from 8 detectors, the trigger was generated by detectors 1 and 5 (triggering occurred in a $16 \mathrm{~ns}$ time window) and as we can see the signals from the detectors are registered with different time delays.

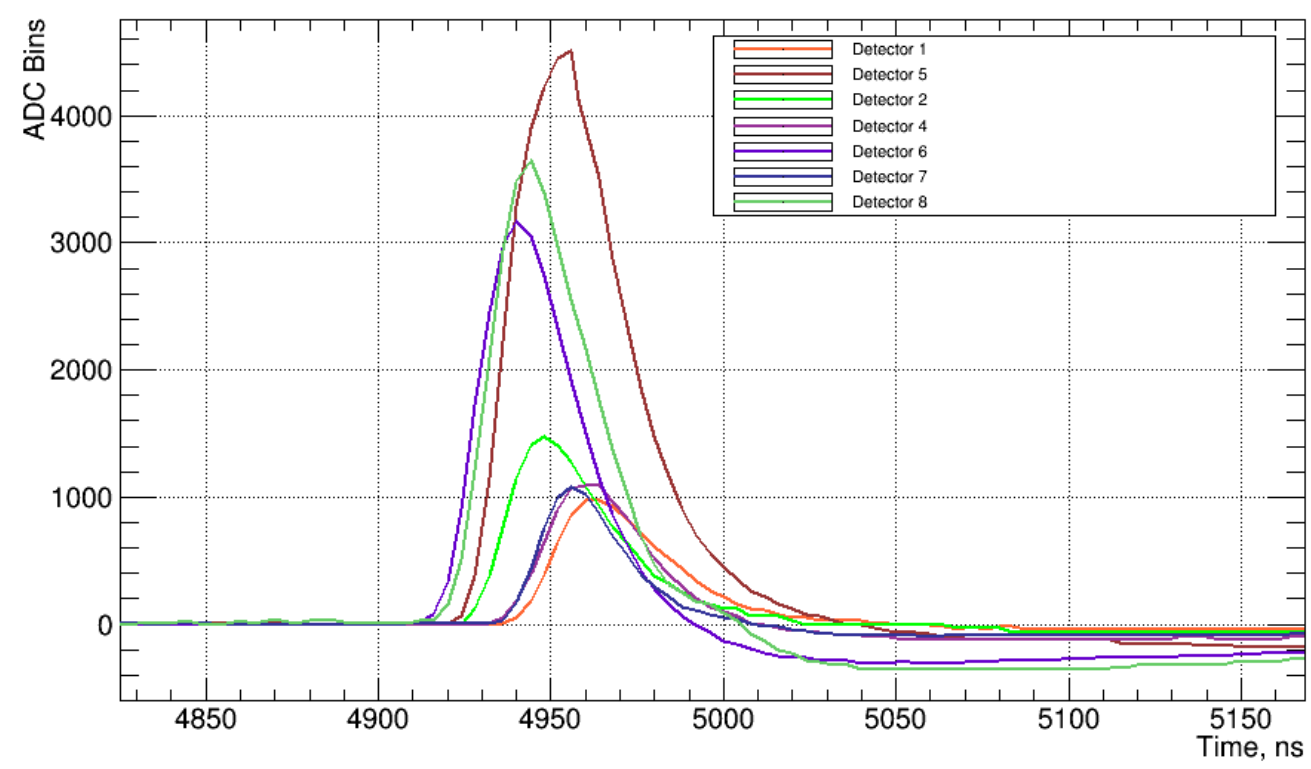

Figure 4 - Preliminary data from the Chronotron

In Figure 4, you can see which detector is activated first by the peak of the amplitude obtained. Table 1 shows (for figure 4 ) the total pulse area and the time value at peak amplitude of each detector. Table 2 shows the delay time of each detector relative to detector 6 . 
Table 1 - Data of single event

\begin{tabular}{|l|c|c|}
\hline Detectors & Time, $n s$ & $\begin{array}{c}\text { Total pulse area, custom } \\
\text { units }\end{array}$ \\
\hline Detector -1 & $4963 \pm 0.4$ & $\mathbf{3 8 4 9 0}$ \\
\hline Detector -2 & $4948 \pm 0.3$ & 54873 \\
\hline Detector -4 & $4958 \pm 0.7$ & 38528 \\
\hline Detector -5 & $4950 \pm 0.4$ & 181792 \\
\hline Detector -6 & $4939 \pm 0.6$ & 106996 \\
\hline Detector -7 & $4955 \pm 0.5$ & $\mathbf{3 1 8 8 6}$ \\
\hline Detector -8 & $4943 \pm 0.8$ & 125912 \\
\hline
\end{tabular}

Table 2 - Delay time of the system

\begin{tabular}{|c|c|c|c|c|c|c|c|}
\hline Detectors & $\mathbf{6}$ & $\mathbf{8}$ & $\mathbf{2}$ & $\mathbf{5}$ & $\mathbf{7}$ & $\mathbf{4}$ & $\mathbf{1}$ \\
\hline Delay, $\mathrm{ns}$ & $\mathbf{0}$ & $\sim 4$ & $\sim 9$ & $\sim 11$ & $\sim 16$ & $\sim 19$ & $\sim 24$ \\
\hline
\end{tabular}

\section{Conclusion}

'Chronotron' setup has been assembled and its now functional. Preliminary data has been acquired and analyzed. The time resolution allows for EAS arrival angle estimation. The accuracy of this measurement will be explored as more data will be available.

\section{Acknowledgment}

This work was supported by the grant from the Committee of Science of the Ministry of Education and Science of the Republic of Kazakhstan AP09058049 "Study of extensive air showers with delayed fronts".

\section{References}

[1] R. Beisembaev et al., Extensive Air Showers with Unusual Spatial and Temporal Structure, in proceedings of EPJ Web of Conferences 208, 06002 (2019)

[2] MELZ-FEU, 4922-y pr-d, 4c5, Zelenograd, g. Moskva, Russia, 124482 (http://www.melz-feu.ru).

[3] CAEN S.p.A. Via della Vetraia, 11, 55049 Viareggio Lucca, Italy. http://caen.it.

[4] N.N. Prokopenko Dissertation for the degree of candidate of physical and mathematical sciences, 2020. 